\title{
PRP in recurrent implantation failure, hope or hype? A prospective randomized controlled study
}

\section{Original Article}

\author{
Kamal Eldin Abdalla Rageh', Ahmed Abdul- Azim Barakat ${ }^{2}$, Khaled Galal Ahmed', \\ Abdullah Mohamed-Ahmed ${ }^{2}$
}

${ }^{I}$ Department of Obstetrics and Gynecology, Faculty of Medicine, ${ }^{3}$ International Islamic Center For Population Studies and Researches, Al-Azhar University, Cairo, Egypt, ${ }^{2} I V F$

Unit, Al-Baraka Fertility Hospital, Bahrain

\begin{abstract}
Introduction: The endometrial function and endometrial receptivity have been accepted to be major limiting factors in the establishment of pregnancy. In spite of improved almost all aspects of IVF: ovarian stimulation, embryo culture and transfer, the pregnancy rates still not satisfactory. The bottleneck is the process of implantation. Recurrent implantation failure (RIF) is one of the nightmares in reproductive medicine and despite several strategies that have been described for management; there is no universal agreement yet. Recently, intrauterine infusion of platelet-rich plasma (PRP) is described to promote endometrial growth and receptivity, PRP has been investigated as a therapeutic approach for several medical disorders in dermatology and rheumatology, but its use in IVF is still limited.

Aim: To evaluate the effectiveness of intrauterine perfusion of autologous platelet-rich plasma in improvement of pregnancy rate in RIF patients.

Design: Prospective randomized controlled study.

Patients and Methods: After ethical committee approval was obtained, 150 infertile women with history of RIF gave their consent to be included in this study, with age below $40 \mathrm{yrs}$, body mass index (BMI) below $30 \mathrm{~kg} / \mathrm{m} 2$. They were divided into 2 comparable groups ; all participants underwent antagonist protocol. In the study group, intrauterine infusion of (PRP) was performed $48 \mathrm{hrs}$ before blastocyst transfer, pregnancy tests were done 12 days after ET.

Results: Out of 75 participants in each group, 32 got pregnant (43\%) in the study group, compared to 11 pregnant participants (15\%) in control group.

Conclusion: According to our study, PRP significantly improved the pregnancy rate and may be a new hope in RIF patients.
\end{abstract}

Key Words: Implantation, IVF, platelet-rich plasma, recurrent implantation failure.

Received: 08 October 2019, Accepted: 10 October 2019

Corresponding Author: Kamal Eldin Abdalla Rageh, M.D., Department of Obstetrics and Gynaecology, Faculty of Medicine, Al-Azhar University, Cairo, Egypt, E-mail: dr_kamal_rageh@yahoo.com.

ISSN: 2090-7265, February 2020, Vol. 10, No. 1

\section{INTRODUCTION}

RIF greatly affects the likelihood of clinical pregnancy after IVF cycles. However, there is little information on its pathogenesis and no consensus among clinicians and researchers on its diagnostic criteria and treatment ${ }^{[1]}$.

It is a distressing condition for patients and frustrating for clinicians and scientists, it is estimated that approximately $10 \%$ of women seeking IVF treatment will experience this particular problem ${ }^{[2]}$.

Despite expanding experience in advanced reproductive technologies and great improvement in infertility treatment, implantation failure is one of the major challenges ${ }^{[3]}$.

The process of implantation involves two main components, a healthy embryo that should have the potential to implant and a receptive endometrium that should enable implantation. The "cross-talk" between the embryo and the endometrium that finally leads to apposition, attachment and invasion of embryos is mandatory for successful implantation and subsequent normal placentation ${ }^{[3]}$.

Recurrent implantation failure is a nightmare for both the patients and the doctor. It needs a wide range of investigations and there is a debate about the lines of management. Several methods have been performed for RIF management but there is little consensus on the most effective one. Blastocyst transfer, preimplantation genetic screening (PGS), assisted hatching, co-culture system, sequential transfer, hysteroscopy, endometrial scratching, salpingectomy for tubal disease, extra number embryo 
transfer, natural cycle, oocyte donation, intra-tubal ET, immune therapy and endometrial receptivity array (ERA) have been used but till now the pregnancy rate after ICSI cycle in RIF patients still unsatisfactory ${ }^{[4]}$.

Intrauterine infusion of platelet-rich plasma (PRP) is a new approach that has been suggested for the treatment of thin endometrium ${ }^{[5]}$. PRP is blood plasma prepared from fresh whole blood that has been enriched with platelets. It is collected from peripheral veins and contains several growth factors such as vascular endothelial growth factor (VEGF), epidermal growth factor (EGF), platelet derived growth factor (PDGF), transforming growth factor (TGF) and other cytokines that stimulate proliferation and growth. Recently, PRP has been used in several medical conditions in ophthalmology, orthopedics, surgery and wound healing but it's efficacy in endometrial growth has not been fully elucidated ${ }^{[6,7,8,9,10,11,12]}$.

\section{AIM OF THE STUDY}

The study aimed to evaluate the effectiveness of intrauterine perfusion of autologous platelet-rich plasma in improvement of pregnancy rate in RIF patients.

\section{MATERIALS AND METHODS}

\section{Study design}

This was a prospective RCT (registered at clinical trials.gov with ID: NCT04058783) which conducted in Al-Baraka Fertility Hospital, Manama, Bahrain. After ethical committee, an approval was obtained, 150 infertile women with history of RIF gave their written consent to be included in this study. All were recruited from the outpatient clinic of Al-Baraka fertility hospital 150 women were included in this study who failed to conceive after 3 or more ET with high-quality embryos were assessed for eligibility to enter the study from July 2018 to March 2019.

The inclusion criteria were age below 40 yrs., body mass index (BMI) below $30 \mathrm{~kg} / \mathrm{m} 2$. The exclusion criteria were hematological and immunological disorders, hormonal disorders, chromosomal and genetic abnormalities and uterine abnormalities (acquired or congenital) as confirmed by HSG and U/S to limit additional factors that may affect the results of the study.

Laboratory evaluation of hormonal, hematological and immunological disorders was done then divided into 2 comparable groups; all participants underwent antagonist protocol, oocytes retrieval, fertilization and embryo transfer; Good quality blastocysts (Grade A or B according to embryologic scoring) transferred for all of the participants.

In study group (75 patients), intrauterine infusion of PRP was done 48 hours before ET. PRP was prepared from autologous blood and it was made by using two steps centrifuge process. All Blastocyst transfers were performed under ultrasound guidance by one expert gynecologist with infertility fellowship. ET was performed according to American Society for Reproductive Medicine (ASRM) guidelines 2013 (Two or three embryos for each participant). On PRP infusion day, $17.5 \mathrm{ml}$ of peripheral venous blood was drawn into a syringe that contains $2.5 \mathrm{ml}$ of Acid Citrate and centrifuged immediately at $1200 \mathrm{rpm}$ for $12 \mathrm{~min}$ to separate red blood cells, then plasma was centrifuged again at $3300 \mathrm{rpm}$ for $7 \mathrm{~min}$ to obtain PRP that contained platelet 4-5 times more than peripheral blood. 0.5- $1 \mathrm{ml}$ of PRP was infused into the uterine cavity with embryo transfer catheter (Wallace - Smiths, UK). On the other side, No PRP was done in control group. Pregnancy tests were done 12 days after ET.

\section{Outcome assessment}

Pregnancy was determined by positive serum $\beta$-HCG, 12 days after ET.

\section{RESULTS}

A total of 150 participants with RIF history were entered into this study. All of them were able to complete the study and their data were analyzed. Table 1 provides baseline characteristics summary.

Participants had a history of failed previous ET attempts between 3-5 times and their mean age was 29.6 \pm 3.7 years.

Table 1: Demographic data of studied patients.

\begin{tabular}{|c|c|c|c|c|c|}
\hline \multirow{2}{*}{ Variables } & Groups & Study & Control & \multicolumn{2}{|c|}{ T-test } \\
\hline & & & & $\mathrm{T}$ & $P$ value \\
\hline \multirow{3}{*}{ Age (years) } & Mean & 29.3 & 29.9 & \multirow{3}{*}{1.1} & \multirow{3}{*}{0.2} \\
\hline & & & & & \\
\hline & $\pm \mathrm{SD}$ & 3.5 & 3.9 & & \\
\hline \multirow{3}{*}{ BMI } & Mean & 26.7 & 26.6 & \multirow{3}{*}{0.5} & \multirow{3}{*}{0.6} \\
\hline & & & & & \\
\hline & $\pm \mathrm{SD}$ & 1.1 & 1.08 & & \\
\hline
\end{tabular}




\begin{tabular}{|c|c|c|c|c|c|}
\hline \multirow{3}{*}{ Infertility duration (years) } & Mean & 6.6 & 6.2 & \multirow{3}{*}{0.6} & \multirow{3}{*}{0.5} \\
\hline & & & & & \\
\hline & $\pm \mathrm{SD}$ & 3.7 & 4.4 & & \\
\hline \multirow{5}{*}{ Cause of infertility } & $\widehat{\partial}$ factor & $53(71 \%)$ & $42(56 \%)$ & \multirow{5}{*}{6.8} & \multirow{5}{*}{0.07} \\
\hline & Tubal factor & $11(15 \%)$ & $21(28 \%)$ & & \\
\hline & & & & & \\
\hline & $\mathrm{PCO}$ & $2(2 \%)$ & $0(0 \%)$ & & \\
\hline & Unexplained & $9(12 \%)$ & $12(16 \%)$ & & \\
\hline \multirow{3}{*}{ AMH (IU/ml) } & Mean & 1.5 & 1.3 & \multirow{3}{*}{0.7} & \multirow{3}{*}{0.4} \\
\hline & & & & & \\
\hline & $\pm \mathrm{SD}$ & 0.8 & 0.7 & & \\
\hline \multirow{3}{*}{ No. of previous failed trials } & Mean & 4.4 & 4.2 & \multirow{3}{*}{0.6} & \multirow{3}{*}{0.6} \\
\hline & & & & & \\
\hline & $\pm \mathrm{SD}$ & 1.1 & 1.1 & & \\
\hline
\end{tabular}

Table 2: Both groups were comparable in the characteristics of IVF cycles as shown in this table

\begin{tabular}{|c|c|c|c|c|c|}
\hline \multirow{2}{*}{ Variables } & \multirow[t]{2}{*}{ Groups } & \multirow{2}{*}{$\begin{array}{l}\text { Study } \\
(\mathrm{N}=75\end{array}$} & \multirow{2}{*}{$\begin{array}{c}\text { Control } \\
(\mathrm{N}=75)\end{array}$} & \multicolumn{2}{|c|}{ T-test } \\
\hline & & & & $\mathrm{T}$ & $P$ value \\
\hline \multirow{3}{*}{ HMG (Amp) } & Mean & 36.7 & 41.1 & & \\
\hline & & & & 1.5 & 0.1 \\
\hline & $\pm \mathrm{SD}$ & 15.1 & 19.3 & & \\
\hline \multirow{3}{*}{$\begin{array}{l}\text { Duration of stimulation } \\
\text { (Days) }\end{array}$} & Mean & 12.7 & 12.4 & & \\
\hline & & & & 0.7 & 0.4 \\
\hline & $\pm \mathrm{SD}$ & 2.4 & 2.7 & & \\
\hline \multirow{3}{*}{$\begin{array}{l}\text { End. thickness } \\
(\mathrm{mm})\end{array}$} & Mean & 10.8 & 10.7 & & \\
\hline & & & & 0.3 & 0.7 \\
\hline & $\pm \mathrm{SD}$ & 1.9 & 2.2 & & \\
\hline \multirow{3}{*}{ Oocyte retrieved } & Mean & 8.9 & 8.2 & & \\
\hline & & & & 2.8 & 0.3 \\
\hline & $\pm \mathrm{SD}$ & 4.2 & 2.8 & & \\
\hline \multirow{3}{*}{ Oocyte fertilized } & Mean & 4.2 & 3.6 & & \\
\hline & & & & 1.8 & 0.06 \\
\hline & $\pm \mathrm{SD}$ & 2.3 & 1.6 & & \\
\hline \multirow{3}{*}{ No. of trans. embryos } & Mean & 2.5 & 2.3 & & \\
\hline & & & & 0.8 & 0.4 \\
\hline & $\pm \mathrm{SD}$ & 0.8 & 0.7 & & \\
\hline
\end{tabular}


Table 3: Comparison between studied groups (study and control) as regards pregnancy test

\begin{tabular}{lcccc}
\hline & Groups & $\begin{array}{c}\text { Study } \\
(\mathrm{N}=75)\end{array}$ & $\begin{array}{c}\text { Control } \\
(\mathrm{N}=75)\end{array}$ & $\begin{array}{c}\text { T-test } \\
\text { Variables }\end{array}$ \\
\hline Pregnancy test & Negative & $43(57 \%)$ & $64(85 \%)$ & $11(15 \%)$ \\
\hline
\end{tabular}

$*$ : $p$-value $<0.001$ is considered highly significant.

Out of 75 participants in each group, 32 got pregnant $(43 \%)$ in the study group, compared to 11 pregnant participants $(15 \%)$ in control group. This table shows highly statistical significant difference $(p$-value $<0.001)$ between studied groups (Study and Control) as regard pregnancy

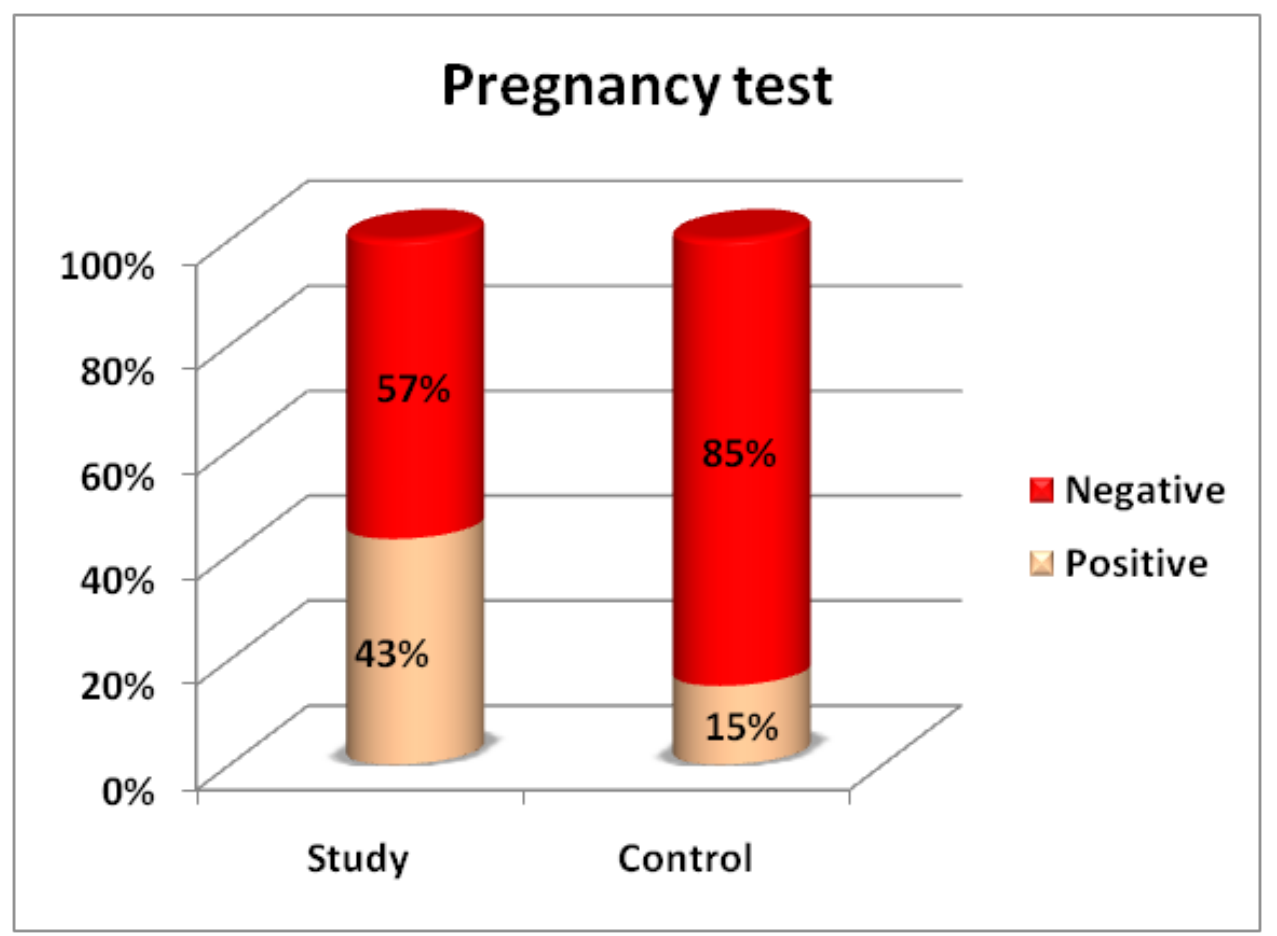

Fig. 1: Comparison between studied groups (Study and Control) as regards the pregnancy test.

\section{DISCUSSION}

\section{Principal Findings}

The result of our study revealed that endometrial perfusion of platelet-rich plasma (PRP) significantly improved the pregnancy rate and maybe a new hope in those patients with a history of recurrent implantation failure (RIF). Out of 75 participants in each group, 32 got pregnant (43\%) in the study group after endometrial PRP infusion, compared to 11 pregnant participants $(15 \%)$ in the control group.

\section{Results of the study in the context of other observations}

Still there is no universally accepted definition for RIF, but most fertility specialists agree that it refers to the failure to achieve a clinical pregnancy after transfer of at least four good-quality embryos in a minimum of three fresh or frozen cycles in a woman under the age of 40

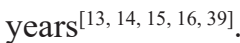

This problem repeats itself and is frequently seen in most IVF units. Even after an IVF cycle in which everything 
apparently goes well, after transfer of apparently good quality 3 embryos the pregnancy rates are around $50 \%$.In contrary, pregnancies could happen after transfer of poor quality single embryo.

From the clinical point of view, it is worthy to note that the term 'implantation failure' refers to two different types of situation, those in whom there has never been evidence of implantation (no detectable HCG production) and those who have evidence of implantation (detectable HCG production) but it did not proceed to beyond the formation of a gestational sac visible on ultrasonography. Implantation failure may be a consequence of embryo or endometrial factors ${ }^{[17]}$.

The endometrial function and endometrial receptivity have been accepted to be major limiting factors in the establishment of pregnancy. In spite of improved almost all aspects of IVF: ovarian stimulation, embryo culture and transfer, the pregnancy rates still not satisfactory ${ }^{[18]}$. The bottleneck is the process of implantation.

Implantation is the process by which the embryo attaches to the uterine wall and first penetrates the epithelium and then the circulatory system of the mother to form the placenta. It's the most vital and the least understood part of reproduction.

The endometrium is normally a non-receptive environment for an embryo except during the window. Implantation window is a period during which the endometrium is optimally receptive to implanting blastocyst within the cycle days 20 and $24^{[19]}$.

The cross-talk between the endometrium and the developing embryo is mediated by many substances, including cytokines (IL1, IL6 and its product LIF), integrins, adhesion molecules, metallo-proteins, growth factors, prostaglandins and hormones such as hCG, all of which support the process of apposition, adhesion, and invasion $^{[20]}$.

Unresponsive endometrium is difficult to treat and obviously contributes to implantation failure. Several approaches have been implemented to increase endometrial receptivity, and presumably prepare it for the "window of implantation". Treatment with high dose oral estrogen or vaginal estradiol application, intended to increase the estradiol level in the serum, as well as in the vicinity of the endometrium, has demonstrated only marginal success $(12,22)$. Similarly, treatment with low dose $\operatorname{aspirin}^{[23]}$ or vaginal sildenafil (24), which presumably increases blood flow to the uterus, consequently improving the response to estradiol, were rather disappointing ${ }^{[25]}$.

RIF may sometimes be associated with a thin endometrium $(<7 \mathrm{~mm})$ noted at the time of ultrasound examination on the day of HCG administration or embryo transfer. The observation suggests that the endometrium is not optimally responding to estrogenic stimulation. From time to time, the underlying cause may not be obvious ${ }^{[26]}$.

Adequate endometrial thickness is the main factor for implantation and pregnancy. Women with persistent thin endometrium often do not undergo embryo transfer. Several methods have been described for endometrial preparation but there is not any definitive method yet. In recent years, intrauterine infusion of G-CSF has been studied but inconsistent results have been reported. Some researchers reported that G-CSF favors endometrial growth and pregnancy. G-CSF is a cytokine that stimulates neutrophilic granulocyte differentiation and proliferation, it may induce endometrium proliferation and growth, thus improve pregnancy outcome ${ }^{[27,28]}$.

Sak and his colleagues investigated that expression of growth factors in the endometrium of women with RIF history is less than normal fertile women ${ }^{[27,29,30,31]}$.

According to this hypothesis local infusion of PRP that contains several growth factors and cytokines may improve endometrial receptivity and implantation.

PRP is autologous blood plasma that has been enriched with platelets at about 4-5 times more than the circulating blood. PRP can stimulate proliferation and regeneration with a large amount of growth factors and cytokines, including PDGF, TGF, VGEF, EGF, fibroblast growth factor (FGF), insulin-like growth factor I, II (IGF I, II), interleukin 8 (IL-8) and connective tissue growth factor (CTGF). Currently, PRP infusion is being increasingly used in several fields in medicine such as nerve injury, osteoarthritis, chronic tendinitis, bone repair and regeneration, cardiac muscles, alopecia, plastic surgery and oral surgery, but there is limited experience in gynecology and

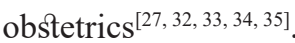

Based on this assumption, local infusion of PRP that contains several growth factors and cytokines may add value. PRP is collected from autologous blood sample, so in comparison to G-CSF, PRP is more accessible and affordable ${ }^{[27,36,37]}$.

For the first time, Chang reported the efficacy of intrauterine infusion of PRP for endometrial growth in women with thin endometrium. In that trial, PRP was infused in 5 women with inadequate endometrium who had a poor response to conventional therapy during the FET cycle. The proper response to treatment was reported in all of them, and normal pregnancy was reported in 4 women ${ }^{[5,27]}$.

Recently, Reghini and co-workers suggested the efficacy of PRP for the treatment of inflammatory response in chronic degenerative endometritis in mares. In this trial, 13 mares with endometrium classified as chronic degenerative endometritis and 8 mares with normal endometrial histology were selected to investigate the PRP therapy effect. The mares were inseminated with fresh semen in two consecutive cycles in a crossover 
study design. Each mare served as its own control and the treatment was performed with intrauterine PRP infusion four hrs after artificial insemination. They concluded that PRP was effective in modulating the exacerbated uterine inflammatory response to semen in mares with chronic degenerative endometritis ${ }^{[27,38]}$.

Just recently, the result of Leila Nazari, et al., a study revealed the efficacy of PRP intrauterine infusion on implantation and pregnancy. In their trial, twenty women with a history of RIF who were candidates for frozen-thawed embryo transfer were recruited in that study. Intrauterine infusion of $0.5 \mathrm{ml}$ of platelet-rich plasma that contained platelet 4-5 times more than peripheral blood sample was performed $48 \mathrm{hrs}$ before blastocyst transfer and eighteen participants were pregnant with one early miscarriage and one molar pregnancy. Sixteen clinical pregnancies were recorded and their pregnancies are ongoing ${ }^{[27]}$.

\section{STRENGTHS AND LIMITATIONS}

The main strengths of our study include: 1) this is by far the first study discussing this topic in our country, as our hospital is the leader IVF center here in Bahrain. 2) The low risk of bias between the study and control group as both of them were statistically comparable. 3) We exclude those patients with hematological and immunological disorders, hormonal disorders, chromosomal and genetic abnormalities and uterine abnormalities (acquired or congenital) as confirmed by HSG and U/S to limit additional factors that may affect the results of the study. 4) All Blastocyst transfers were performed under ultrasound guidance by only one expert gynecologist with infertility fellowship. 5) The use of individual patient data for direct comparison between both groups. 6) The consistency between our results and those of the previous trials in the literature.

Some limitations have to be noticed, as we are living here in a small country so, the sample size may need to be increased in the further coming clinical trials, also at the time of embryo transfer we didn't select those embryos based on a genetic basis as we are usually not doing PGT as a routine; so we select the embryos phenotypically according to the embryo scoring.

\section{CONCLUSION}

The recurrent implantation failure is hard to be managed, and if all available treatments fail, then endometrial PRP can add value. The local infusion of PRP that contains several growth factors and cytokines may improve endometrial receptivity and implantation. PRP is collected from autologous blood sample, so in comparison to G-CSF, PRP is more accessible and affordable.

PRP can stimulate proliferation and regeneration with a large amount of growth factors and cytokines. Currently,
PRP infusion is being increasingly used in several fields in medicine but still not widely applied in infertility management.

In conclusion: The result of our study revealed that platelet-rich plasma significantly improved the pregnancy rate and maybe a new hope in RIF patients. Out of 75 participants in each group, 32 got pregnant $(43 \%)$ in the study group after endometrial PRP infusion, compared to 11 pregnant participants $(15 \%)$ in the control group.

\section{CONFLICTS OF INTEREST}

There are no conflicts of interest.

\section{REFERENCES}

1. Feng Guo, Ming-Juan Zhou, Ai-Jun Zhang, Advances in the Treatment of Recurrent Implantation Failure Reproductive and Developmental Medicine : June 25, 2017 Volume 1 ; Issue 2.

2. C. Coughlan, W. Ledger, Q. Wang, Fenghua Liu, Aygul Demirol, Timur Gurgan, R. Cutting, K. Ong, H. Sallam, T.C. Li' Correspondence information about the author T.C. Li, Recurrent implantation failure: definition and management, Reproductive BioMedicine Online (2014) 28, 14- 38.

3. Alex Simon and Neri Laufer, Assessment and treatment of repeated implantation failure (RIF), J Assist Reprod Genet. 2012 Nov; 29(11): 1227-1239.

4. Tanya Timeva, Atanas Shterev and Stanimir Kyurkchiev: Recurrent Implantation Failure: The Role of the Endometrium, J Reprod Infertil. 2014 Oct-Dec; 15(4): 173-183.

5. Chang Y, Li J, Chen Y, Wei L, Yang X, Shi Y, et al. Autologous platelet-rich plasma promotes endometrial growth and improves pregnancy outcome during in vitro fertilization. Int $\mathrm{J}$ Clin Exp Med. 2015; 8:1286-1290. [PMC free article] [PubMed].

6. El-Anwar MW, Nofal AA, Khalifa M, Quriba AS, Use of autologous platelet-rich plasma in complete cleft palate repair. $2016 \mathrm{Jul} ; 126(7): 1524-8$. doi: 10.1002/lary.25868. Epub 2016 Apr 14. [PubMed].

7. Lee HR, Shon OJ, Park SI, Kim HJ, Kim S, Ahn MW, Do SH. Platelet-Rich Plasma Increases the Levels of Catabolic Molecules and Cellular Dedifferentiation in the Meniscus of a Rabbit 
Model. Int J Mol Sci. 2016 Jan 16;17(1). pii: E120. doi: 10.3390/ijms17010120. [PMC free article] [PubMed].

8. Maria-Angeliki G, Alexandros-Efstratios K, Dimitris R, Konstantinos K. Platelet-rich Plasma as a Potential Treatment for Noncicatricial Alopecias. Int J Trichol. 2015; 7:54-63. [PMC free article] [PubMed].

9. Picard F1, Hersant B1, Bosc R1, Meningaud JP1, The growing evidence for the use of platelet-rich plasma on diabetic chronic wounds: A review and a proposal for a new standard care. Sep;23(5):63843. doi: 10.1111/wrr.12317. Epub 2015 Aug 25. [PubMed].

10. Corrado Ronci, Angelo Salvatore Ferraro, Alessandro Lanti, Filippo Missiroli, Silvia Sinopoli, Gianpaolo Del Proposto, Chiara Cipriani, Cecilia De Felici, Federico Ricci, Marco Ciotti, Laura Cudillo, William Arcese, Gaspare Adorno., Platelet-rich plasma as treatment for persistent ocular epithelial defects 2015; June 2015 Volume 52, Issue 3, Pages 300-304.

11. Rossi LA, Molina Romoli AR, Bertona Altieri BA, Burgos Flor JA, Scordo WE, Elizondo CM. Does platelet-rich plasma decrease time to return to sports in acute muscle tear? A randomized controlled trial.Knee Surg Sports Traumatol Arthrosc. 2016 [PubMed].

12. Sadabad HN, Behzadifar M, Arasteh F, Behzadifar M, Dehghan HR. Efficacy of Platelet-Rich Plasma versus Hyaluronic Acid for treatment of Knee Osteoarthritis: A systematic review and metaanalysis. Electron Physic. 2016; 8:2115-2022. [PMC free article] [PubMed].

13. Das, M., Holzer, H.E., 2012. Recurrent implantation failure: gamete and embryo factors. Fertil. Steril. 97, 1021-1027.

14. Laufer, N., Simon, A., 2012. Recurrent implantation failure: current update and clinical approach to an ongoing challenge. Fertil. Steril. 97, 1019-1020.

15. Penzias AS , Recurrent IVF failure: other factors, 2012 May; 97(5):1033-8. doi: 10.1016/j.fert. stert.2012.03.017. Epub 2012 Mar 28.

16. Urman, B., Yakin, K., Balaban, B., 2005. Recurrent implantation failure in assisted reproduction: how to counsel and manage. B. Treatment options that have not been proven to benefit the couple. Reprod. Biomed. Online 11, 382-391.

17. Coughlan C, Ledger W, Wang Q, Liu F, Demirol A, Gurgan T, et al. Recurrent implantation failure: definition and management. Reprod Biomed Online. 2014; 28:14-38. [PubMed].

18. Tanya Timeva, Atanas Shterev and Stanimir Kyurkchiev, Recurrent Implantation Failure: The Role of the Endometrium, J Reprod Infertil. 2014 Oct-Dec; 15(4): 173-183.

19. Alok Sharma and Pratap Kumar, Understanding implantation window, a crucial phenomenon, J Hum Reprod Sci. 2012 Jan-Apr; 5(1): 2-6.

20. Su-Mi Kim and Jong-Soo Kim, A Review of Mechanisms of Implantation, Dev Reprod. 2017 Dec; 21(4): 351-359.

21. Check JH. The importance of sonographic endometrial parameters in influencing success following embryo transfer in the modern era and therapeutic options - part 1: the importance of late proliferative phase endometrial thickness. Clin Exp Obstet Gynecol. 2011;38:197-200. doi: 10.1016/S0889-8545(11)00020-9. [PubMed] [Cross Ref].

22. Tourgeman DE, Slater CC, Stanczyk FZ, Paulson RJ. Endocrine and clinical effects of micronized estradiol administered vaginally or orally. Fertil Steril. 2001; 75:200-202. doi: 10.1016/S00150282(00)01640-X. [PubMed] [Cross Ref].

23. Weckstein LN, Jacobson A, Galen D, Hampton K, Hammel J. Low-dose aspirin for oocyte donation recipients with a thin endometrium: prospective, randomized study. Fertil Steril. 1997; 68:927-930. doi: 10.1016/S0015-0282(97)00330-0. [PubMed] [Cross Ref].

24. Sher G, Fisch JD. Effect of vaginal sildenafil on the outcome of in vitro fertilization (IVF) after multiple IVF failures attributed to poor endometrial development. Fertil Steril. 2002; 78:1073-1076. doi: 10.1016/S0015-0282(02)03375-7. [PubMed] [Cross Ref].

25. Senturk LM, Erel CT. Thin endometrium in assisted reproductive technology. Curr Opin 
Obstet Gynecol. 2008; 20:221-228. doi: 10.1097/ GCO.0b013e328302143c. [PubMed] [Cross Ref].

26. Tao Zhang, MMed, Zhou Li, PhD, Xinling Ren, $\mathrm{PhD}$, Bo Huang, PhD, Guijin Zhu, MMed, Wei Yang, MMed, and Lei Jin, PhD, Endometrial thickness as a predictor of the reproductive outcomes in fresh and frozen embryo transfer cycles, Medicine (Baltimore). 2018 Jan; 97(4): e9689.

27. Leila Nazari, Saghar Salehpour, Nasrin Saharkhiz and Shahrzad Zadehmodarres, Treatment of thin endometrium with autologous platelet-rich plasma: a pilot study, JBRA Assist Reprod. 2017 Jan-Mar; 21(1): 54-56.

28. Aflatoonian N, Eftekhar M, Aflatoonian B, Rahmani E, Aflatoonian A. Surrogacy as a good option for treatment of repeated implantation failure: a case series. Iran J Reprod Med. 2013;11:77-80.[PMC free article] [PubMed].

29. Sak ME, Gul T, Evsen MS, Soydinc HE, Sak $\mathrm{S}$, Ozler A, et al. Fibroblast growth factor-1 expression in the endometrium of patients with repeated implantation failure after in vitro fertilization. Eur Rev Med Pharmacol Sci. 2013; 17:398-402. [PubMed].

30. Granot I, Gnainsky Y, Dekel N. Endometrial inflammation and effect on implantation improvement and pregnancy outcome. Reproduction. 2012; 144:661-668. [PubMed].

31. Wang H, Dey SK. Roadmap to embryo implantation: clues from mouse models. Nat Rev Genet. 2006; 7:185-199. [PubMed].

32. Alcaraz J, Oliver A, Sanchez JM. Platelet-Rich Plasma in a Patient with Cerebral Palsy. Am J
Case Reports. 2015; 16:469-472. [PMC free article] [PubMed].

33. Borrione $\mathrm{P}$, Gianfrancesco AD, Pereira MT, Pigozzi F. Platelet-rich plasma in muscle healing. Am J Physic Med Rehabilit. 2010; 89:854-861. [PubMed].

34. Patel AN, Selzman CH, Kumpati GS, McKellar $\mathrm{SH}$, Bull DA. Evaluation of autologous platelet rich plasma for cardiac surgery: outcome analysis of 2000 patients. J Cardiothorac Surg. 2016; 11:62. [PMC free article] [PubMed].

35. Yu W, Wang J, Yin J. Platelet-rich plasma: a promising product for treatment of peripheral nerve regeneration after nerve injury. Int $\mathrm{J}$ Neurosci. 2011; 121:176-180. [PubMed].

36. Gleicher N, Vidali A, Barad DH. Successful treatment of unresponsive thin endometrium. Fertil. Steril. 2011;95(2123):e13-e17. [PubMed].

37. Elkin Lucena and Harold Moreno-Ortiz, Granulocyte colony-stimulating factor (G-CSF): a mediator in endometrial receptivity for a patient with polycystic ovary (PCO) undergoing in vitro maturation (IVM), BMJ Case Reports 2013; doi:10.1136/bcr-2012-008115.

38. Reghini MF, Ramires Neto C, Segabinazzi LG, Castro Chaves MM, Dell'Aqua Cde P, Bussiere $\mathrm{MC}$, et al. Inflammatory response in chronic degenerative endometritis mares treated with platelet-rich plasma.Theriogenology. 2016;86:516-522. [PubMed].

39. El-Toukhy T, Taranissi M. Towards better quality research in recurrent implantation failure: standardizing its definition is the first step. Reprod Biomed Online. 2006; 12:383-385. 777[PubMed]. 